\title{
Postpartum depressive symptoms in the first 17 months after childbirth: the impact of an emotionally supportive partnership
}

\author{
Daniela Bielinski-Blattmann · Sakari Lemola • \\ Chantal Jaussi · Werner Stadlmayr • \\ Alexander Grob
}

Received: 12 May 2008/Revised: 19 March 2009/ Accepted: 26 March 2009/Published online: 28 July 2009 (c) Birkhäuser Verlag, Basel/Switzerland 2009

\begin{abstract}
Objectives This study investigates the impact on different postpartum depressive trajectories (i.e., "non depressive symptoms", "stable depressive symptoms", "deterioration" and "improvement") from 5-17 months after childbirth exerted by emotional support that mothers receive from their partners and emotional support they provide to their partners. Methods Postpartum depressive symptoms were assessed using the Edinburgh Postnatal Depression Scale 5 and 17 months after delivery in a sample of 293 mothers. Emotional support received from the partners was assessed among both mothers and partners.

Results The initial level and the change in emotional support that mothers received from their partners were related to different trajectories of postpartum depressive symptoms. Mothers who were living in a partnership with low reciprocal emotional support showed a significantly
\end{abstract}

D. Bielinski-Blattmann · C. Jaussi

Department of Clinical Research, University of Bern,

Bern, Switzerland

S. Lemola · A. Grob

Department of Personality and Developmental Psychology,

University of Basel, Basel, Switzerland

S. Lemola

Department of Psychology, University of Helsinki,

Helsinki, Finland

W. Stadlmayr

Department of Obstetrics and Gynecology,

University Hospital Bern, Bern, Switzerland

D. Bielinski-Blattmann $(\square)$

Frauenklinik des Inselspitals, Universitätsklinik,

Effingerstrasse 102, 3010 Bern, Switzerland

e-mail: daniela.bielinski@dkf.unibe.ch higher risk of suffering from "stable depressive symptoms" than mothers who were living in a partnership with high reciprocal emotional support.

Conclusions An increased risk of persistent depressive symptoms beyond the early postpartum period was observed in mothers with poor reciprocal emotional support in the partnership. Further research is needed for a better understanding of the mothers persistent depressive symptoms after childbirth associated with reciprocity of emotional support in the partnership.

Keywords Postpartum women .

Trajectories of symptoms of postnatal depression .

Social support · Partnership after childbirth

\section{Introduction}

Approximately $13 \%$ of women experience post partum depression (PPD) during the first year after delivery (O'Hara and Swain 1996). PPD is characterized by the whole spectrum of depressive symptoms the most common being feelings of insufficiency and lack of positive feelings for the child, as well as feelings and impulses of compulsion (Rohde 2001). Whereas PPD symptoms disappear in most women during the first months after childbirth, some women suffer from PPD symptoms for several months or even years (Goodman 2004). The development of PPD is significantly related to a lack of social support, particularly emotional support in the partner relationship (Beck 2001; Boyce and Hickey 2005; Lemola et al. 2007). Mothers who receive higher levels of support from their partners report more subjective well-being, better adjustment to motherhood, and lower levels of depressive symptoms (Cox et al. 1989; Eberhard-Gran et al. 2002; Merchant et al. 1995; Patel et al. 2002). 
Up to the present, most research efforts have focused solely on the effect of the support mothers receive, and thus ignored the social support received and provided in systems of social relationships. There are various socio-cognitive theories that make differing predictions on how support in social relationships impacts on the well-being of the individuals in the relationship. From the theory of self-efficacy it can be derived that the support one provides improves the perception of self-efficacy by proving that one is a competent and caring person and thus has mainly a positive effect on the provider (Bandura 1986). Similarly, the mood repair model proposes that supporting others is even motivated by engendering a positive mood state (Cialdini et al. 1973). Thus, support provided to another person should have a positive effect on the well-being of the provider of the support and should relieve him or her from negative feelings. On the other hand, theories on the equity of human behavior imply that it is neither the reception nor the provision of support that improves well-being but rather equitable, reciprocal support in a relationship (Walster et al. 1978). Therefore, research has combined the providing and the receiving dimensions of support in social relationships into a model which proposes four support categories in which individuals can be classified (Maton 1987; Stevenson et al. 1999). These four categories include "bidirectionals", "providers", "receivers", and "low supporters". "Bidirectionals" report high levels of both providing and receiving support. "Providers" are high on providing but low on receiving support, whereas "receivers" are low on providing but high on receiving support. "Low supporters" report low levels of providing and receiving support. The traditional view on social support (Cohan and Wills 1985) predicts high levels of well-being among "bidirectionals" and "receivers" and low levels of well-being among "low supporters" and "providers".

The aim of this study is to investigate whether the different trajectories of postpartum depressive symptoms can be predicted by (a) the initial level of emotional support the mothers receive from their partners after childbirth and (b) the longitudinal change in this received emotional support. Furthermore, the predictions derived from the traditional view on social support and the predictions from the different socio-cognitive theories are examined. The study focuses on the impact of reciprocity, of giving and receiving emotional support in the partnership, with regard to the trajectories of maternal postpartum depressive symptoms.

\section{Methods}

This study was part of a longitudinal study designed to investigate predictors of psychological adaptation in mothers and their partners in an unselected sample. Details of the research protocol and of the recruitment of the sample are described elsewhere (Lemola and Grob 2008).

Participants were recruited after the announcement of a child's birth in a newspaper or on a hospital website. Six weeks after giving birth, 458 women were assessed on several background variables and on perceived emotional support from their partner. The sample was representative for the population of childbearing mothers in Switzerland regarding birth weight (Drack et al. 1998), rate of Cesarean deliveries and parity (Swiss Federal Office of Statistics 2005a, b) but the participating women were slightly older than average and somewhat more highly educated (Swiss Federal Office of Statistics 2005c). Five months after birth, 374 women from the initial sample completed questionnaires on their psychological adjustment (retention rate $=81.6 \%$ ). Seventeen months after childbirth, 293 women completed questionnaires on their psychological adjustment and emotional support from their partner (retention rate $=64.0 \%$ ). The attrition was selective regarding postnatal depression but not selective regarding emotional support from the partner. Mothers who had elevated depression scores [10 points or higher on the Edinburgh Postnatal Depression Scale (EPDS)] 5 months after birth were more likely to drop out before month 17 than mothers with lower scores $\quad($ Retention month5-month17(EPDS $\geq 10)=$ $68.5 \%, \quad$ Retention month5-month $17(\mathrm{EPDS}<10)=80.7 \%, \quad U=$ $10,522.0, P<0.05)$. The sample characteristics at 5 months after childbirth, when postpartum depressive symptoms were assessed for the first time, are presented in Table 1.

Postpartum depressive symptoms as dependent variables

Postpartum depressive symptoms were assessed 5 and 17 months after childbirth with the EPDS (Cox et al. 1998; for German translation see Bergant et al. 1998). The EPDS is a 10-item scale specifically designed to screen postpartum mothers for depression with established validity and reliability (Lee et al. 2002). The items of the EPDS ask the mothers whether, during the previous 7 days, they have suffered from symptoms of depression such as depressed mood, feelings of guilt, anxiety, and suicidal thoughts. The reliability estimates in the present study were good (Cronbach's $\alpha_{\text {month5 }}=0.84$, Cronbach's $\alpha_{\text {month17 }}=0.86$ ). The mean scores for symptoms of depression over the course from 5-17 months postpartum were not significantly different $\left(t_{(293)}=-1.36, P=0.173\right)$.

Assignment of participants to trajectories of postpartum depressive symptoms

In order to reflect the state of depressive symptoms 5 months and the change up to 17 months after childbirth, 
Table 1 Characteristics of the study participants

\begin{tabular}{|c|c|c|c|c|}
\hline & \multicolumn{4}{|c|}{ Reciprocal support groups 5 months postpartum } \\
\hline & $\begin{array}{l}\text { Bidirectional high } \\
\text { supporters }\end{array}$ & Providers & Receivers & $\begin{array}{l}\text { Bidirectional low } \\
\text { supporters }\end{array}$ \\
\hline$N^{\mathrm{a}}(\%)$ & $101(32.1 \%)$ & $61(19.4 \%)$ & $47(14.9 \%)$ & $106(33.7 \%)$ \\
\hline \multicolumn{5}{|l|}{ Demographic variables } \\
\hline \multicolumn{5}{|l|}{ Language region } \\
\hline German $(\%)$ & $66(65.3 \%)$ & $46(75.4 \%)$ & $36(76.6 \%)$ & $76(71.7 \%)$ \\
\hline French $(\%)$ & $35(34.7 \%)$ & $15(24.6 \%)$ & $11(23.4 \%)$ & $30(28.3 \%)$ \\
\hline \multicolumn{5}{|l|}{ Maternal age in years ${ }^{\mathrm{b}}$} \\
\hline Mean (SD) & $32.21(4.07)$ & $31.39(4.01)$ & $32.70(4.39)$ & $33.30(3.96)$ \\
\hline \multicolumn{5}{|l|}{ Education $^{\mathrm{c}}$} \\
\hline Secondary I (\%) & $7(6.9 \%)$ & $2(3.3 \%)$ & $3(6.4 \%)$ & $7(6.6 \%)$ \\
\hline Secondary II (\%) & $67(66.3 \%)$ & $45(73.7 \%)$ & $24(51.0 \%)$ & $65(61.3 \%)$ \\
\hline Tertiary $(\%)$ & $27(26.8 \%)$ & $14(23.0 \%)$ & $20(42.6 \%)$ & $34(32.1 \%)$ \\
\hline Married/cohabiting (\%) & $99(98.0 \%)$ & $61(100 \%)$ & $44(93.6 \%)$ & $103(99.0 \%)$ \\
\hline Duration of the relationship: 2 years or longer (\%) & $97(9.6 \%)$ & $60(98.4 \%)$ & $45(97.8 \%)$ & $103(98.1 \%)$ \\
\hline Depressive symptoms 5 months PPD [Mean (SD)] ${ }^{\mathrm{d}}$ & $4.52(3.99)$ & $4.42(3.59)$ & $4.07(3.35)$ & $7.86(5.21)$ \\
\hline Depressive symptoms 17 months PPD [Mean $(\mathrm{SD})]^{\mathrm{d}}$ & $4.59(4.08)$ & $6.16(4.07)$ & $4.05(3.99)$ & $7.52(4.92)$ \\
\hline
\end{tabular}

${ }^{a} N$ varied because of missing values

${ }^{\mathrm{b}}$ On average, childbearing mothers in Switzerland are 31.0 years old (Swiss Federal Office of Statistics 2005b)

c Among the female population between the ages of 25 and 39 years in Switzerland, 13.5\% have completed secondary education I (i.e., elementary schooling), 59.4\% secondary education II (i.e., finished apprenticeship or high school diploma) and $27.1 \%$ tertiary education (degree from tertiary institution or university degree; Swiss Federal Office of Statistics 2005c)

d EPDS-score: 0-30

the participants were assigned to trajectories of postpartum depressive symptoms. Applying the EPDS cutoff of 9/10 (Cox et al. 1996) and recombining the group memberships at both times four trajectory groups were constructed, "nondepressive symptoms" $\left(\right.$ EPDS $_{\text {month5 }}<10$ and EPDS $_{\text {month17 }}<10 ; \quad n=219 ; 74.7 \%$ ), “improvement" $\left(\right.$ EPDS $_{\text {month5 }} \geq 10$ and EPDS month $17_{17}<10 ; n=23 ; 7.8 \%$ ), "deterioration" $\left(\mathrm{EPDS}_{\text {month5 }}<10\right.$ and $\mathrm{EPDS}_{\text {month17 }} \geq 10$; $n=23 ; 7.8 \%$ ), and "stable depressive symptoms" $\left(\mathrm{EPDS}_{\text {month5 }} \geq 10\right.$ and $\left.\mathrm{EPDS}_{\text {month17 }} \geq 10 ; n=28 ; 9.6 \%\right)$.

Emotional support mothers received and provided

The mothers reported on the emotional support they received from their partners 6 weeks and 17 months after childbirth on a scale containing seven 6-point Likert-type items. This scale measures confidence in being supported by the partner and being able to discuss concerns with him without feeling criticized or rejected. The scale was derived from a research questionnaire on emotional support (Life Course and Health Research Center 2001). The reliability of the scale was good, Cronbach's $\alpha_{\text {week6 }}=0.86$, Cronbach's $\alpha_{\text {month17 }}=0.88$, respectively. The mean scores for this received emotional support were significantly higher 6 weeks after childbirth than 17 months postpartum $\left(t_{(292)}=6.76, P<0.001\right)$.

The partners reported on the emotional support they received from their wives 5 months after childbirth on the same 7-item scale (Cronbach's $\alpha_{\text {month5 }}=0.82$ ). Thus, both partners reported on the emotional support they received from the other.

Assignment of participants to groups of reciprocal support

In order to reflect the exchange of emotional support in the partnership the participants were assigned to support categories. The reciprocal emotional partner support 5 months after childbirth was defined by the emotional support mothers received 6 weeks and partners received 5 months postpartum. Moderate correlations between maternal and paternal reports of the exchange of emotional support 5 months postpartum were found $\left(r_{\text {month } 5}=0.43\right.$, $P<0.001)$.

Mothers were classified into four support groups of reciprocal support 5 months after childbirth: (a) "bidirectional high supporters" (both mothers and partners reported emotional support levels above the median; (b) 
"bidirectional low supporters" (both mothers and partners reported emotional support levels below the median); "receivers" (mothers' providing levels were below the median but their receiving levels were above the median) and d) "providers" (mothers' providing levels were above the median but their receiving levels were below the median). There were $101(32.1 \%)$ "bidirectional high supporters", 61 (19.4\%) "providers", 47 (14.9\%) "receivers" and 106 (33.7\%) "bidirectional low supporters".

\section{Statistical analysis}

All statistical analyses were performed using the SPSS statistical package version 15.0. After examination of the descriptive statistics, binary logistic regressions were computed to predict the trajectories of maternal postpartum depressive symptoms from the emotional support they received from their partners 6 weeks after childbirth and by the longitudinal change of the received emotional support. Logistic regressions were then computed to predict the trajectories of maternal postpartum depressive symptoms from the reciprocal emotional support mothers and their partners exchanged in the partnership 5 months after childbirth.

\section{Results}

Incidence of depressive symptoms 6 and 17 months postpartum

Five months after childbirth 34 mothers (9.1\%) scored above the EPDS cutoff for probable major depression $(\geq 13)$ and 39 mothers $(10.4 \%)$ scored within the range of probable minor depression (10-12) (Cox et al. 1993). Seventeen months after childbirth 27 mothers $(8.4 \%)$ reported probable major depression, whereas 33 mothers (10.2\%) reported probable minor depression. The mean scores of the EPDS did not change significantly between 5 and 17 months postpartum $\left(t_{(293)}=-1.36, P=0.173\right)$. For the further analyses the groups of probable major depression and probable minor depression were combined and are referred to as "postpartum depressive symptoms". Table 2 displays mean values of emotional support the mothers received from their partners 6 weeks and 17 months after childbirth and the longitudinal change of this support for the four trajectories.

Prediction of the different depressive symptom trajectories by emotional support from the partner 6 weeks after childbirth and by the change in this support up to 17 months

Table 3 displays the binary logistic regression models for the different trajectories of postpartum depressive symptoms (Schmidt 2008).

The initial level of emotional support from the partners 6 weeks after childbirth proved to be a significant predictor of the "stable depressive symptom" trajectory. For mothers with lower levels of this initial emotional support the odds of being in the "stable depressive symptom" trajectory were 2.77 times higher than in the "nondepressive symptom" trajectory $(\mathrm{OR}=0.36 ; 95 \% \quad \mathrm{CI}, \quad 0.24-0.56)$. Furthermore, a low level of initial emotional support was associated with twice the odds of being in the "stable depressive symptom" trajectory compared with the "deterioration" trajectory $(\mathrm{OR}=0.50,95 \% \mathrm{CI}, 0.25-1.00)$. Additionally, a change in emotional support from the partner between 6 weeks and 17 months after childbirth

Table 2 Emotional support mothers received from their partners in four trajectories of postpartum depressive symptoms

\begin{tabular}{|c|c|c|c|}
\hline \multirow[t]{2}{*}{ Trajectories of postpartum depression } & \multicolumn{3}{|c|}{$\begin{array}{l}\text { Emotional support mothers received from their partners } \\
\text { (z-scores) }\end{array}$} \\
\hline & $\begin{array}{l}6 \text { weeks post partum } \\
\text { [means }(\mathrm{SD})]\end{array}$ & $\begin{array}{l}17 \text { months post partum } \\
\text { [means (SD)] }\end{array}$ & $\begin{array}{l}\text { Change between } \\
\mathrm{t} 1 \text { and } \mathrm{t}^{\mathrm{a}} \text { [means (SD)] }\end{array}$ \\
\hline "Non depressive symptoms" $(n=219)$ & $0.14(0.06)^{\mathrm{b}}$ & $0.17(0.06)^{\mathrm{b}, \mathrm{c}}$ & $0.03(0.05)^{\mathrm{b}}$ \\
\hline "Improvement" $(n=23)$ & $-0.10(0.18)$ & $0.24(0.19)^{\mathrm{d}}$ & $0.35(0.15)^{\mathrm{c}}$ \\
\hline "Deterioration" $(n=23)$ & $0.02(0.18)^{\mathrm{c}}$ & $-0.42(0.19)^{\mathrm{b}}$ & $-0.44(0.15)^{\mathrm{b}, \mathrm{c}}$ \\
\hline "Stable depressive symptoms" $(n=28)$ & $-0.77(0.17)^{\mathrm{b}, \mathrm{c}}$ & $-0.71(0.17)^{\mathrm{c}, \mathrm{d}}$ & $0.06(0.14)$ \\
\hline
\end{tabular}


Table 3 Results from separate binary logistic regressions predicting different depressive symptom trajectories by emotional support from the partner 6 weeks after childbirth and by the change in this support up to 17 months after childbirth

\begin{tabular}{|c|c|c|c|}
\hline & \multicolumn{3}{|c|}{ Standardized coefficients } \\
\hline & $B$ & OR $(95 \% \mathrm{Cl})$ & $P$ \\
\hline \multicolumn{4}{|l|}{ "Nondepressive symptoms" " versus "Deterioration" ${ }^{\mathrm{e}}$ " } \\
\hline Emotional support mothers received 6 weeks postpartum ${ }^{\mathrm{a}}$ & -0.45 & $0.64(0.38-1.09)$ & \\
\hline Change in emotional support mothers received from the partners ${ }^{\mathrm{a}, \mathrm{b}}$ & -0.95 & $0.38(0.20-0.69)$ & $* * *$ \\
\hline \multicolumn{4}{|l|}{ "Non depressive symptoms ${ }^{\mathrm{c}}$ " versus "Improvement ${ }^{\mathrm{d}}$, } \\
\hline Emotional support mothers received 6 weeks postpartum ${ }^{\mathrm{a}}$ & -0.115 & $0.89(0.52-1.51)$ & \\
\hline Change in emotional support mothers received from the partners ${ }^{\mathrm{a}, \mathrm{b}}$ & 0.55 & $1.74(0.89-3.40)$ & \\
\hline \multicolumn{4}{|l|}{ "Non depressive symptoms" ${ }^{\mathrm{c}}$ " versus "Stable depressive symptoms ${ }^{\mathrm{f}}$} \\
\hline Emotional support mothers received 6 weeks postpartum ${ }^{\mathrm{a}}$ & -1.01 & $0.36(0.24-0.56)$ & $* * *$ \\
\hline Change in emotional support mothers received from the partners ${ }^{\mathrm{a}, \mathrm{b}}$ & -0.47 & $0.63(0.36-1.11)$ & \\
\hline \multicolumn{4}{|l|}{ "Improvement ${ }^{\mathrm{d}}$ " versus "Deterioration ${ }^{\mathrm{e}} "$} \\
\hline Emotional support mothers received 6 weeks postpartum ${ }^{\mathrm{a}}$ & -0.30 & $0.74(0.33-1.68)$ & \\
\hline Change in emotional support mothers received from the partners ${ }^{\mathrm{a}, \mathrm{b}}$ & -1.90 & $0.15(0.04-0.58)$ & $* *$ \\
\hline \multicolumn{4}{|l|}{ "Improvement ${ }^{\mathrm{d}}$ " versus "Stable depressive symptoms" ${ }^{\mathrm{f}}$ ", } \\
\hline Emotional support mothers received 6 weeks postpartum ${ }^{\mathrm{a}}$ & -0.91 & $0.40(0.20-0.80)$ & $*$ \\
\hline Change in emotional support mothers received from the partners ${ }^{\mathrm{a}, \mathrm{b}}$ & -0.94 & $0.39(0.15-0.99)$ & $*$ \\
\hline \multicolumn{4}{|l|}{ "Deterioration" ${ }^{\mathrm{e}}$ versus "Stable depressive symptoms" } \\
\hline Emotional support mothers received 6 weeks postpartum ${ }^{\mathrm{a}}$ & -0.69 & $0.50(0.25-1.00)$ & * \\
\hline Change in emotional support mothers received from the partners ${ }^{\mathrm{a}, \mathrm{b}}$ & 0.53 & $1.70(0.74-3.87)$ & \\
\hline
\end{tabular}

was a significant predictor. A decline in emotional support between 6 weeks and 17 months after childbirth decreased the mother's chances of being in the "nondepressive symptom" compared with the "deterioration" trajectory $(\mathrm{OR}=0.38 ; 95 \%$ CI, 0.20-0.69). Furthermore, mothers who reported high levels of emotional support received 6 weeks after childbirth but whose emotional support declined between 6 weeks and 17 months after childbirth had more than six times higher odds of being in the "deterioration" compared with the "improvement" trajectory $(\mathrm{OR}=0.15 ; 95 \% \mathrm{CI}, 0.04-0.58)$. In addition, both the initial level of emotional support received from the partner 6 weeks after childbirth and the change in emotional support between 6 weeks and 17 months after childbirth were significant predictors for mothers with "stable depressive symptom" trajectory. Mothers with lower levels of initial emotional support or whose emotional support declined were more than two and a half times as likely to belong to the "stable depressive symptom" trajectory than to the "improvement" trajectory $(\mathrm{OR}=0.40 ; 95 \% \mathrm{CI}, 0.20-0.80$ and $\mathrm{OR}=0.39 ; 95 \% \mathrm{CI}$, $0.15-0.99)$. The initial level of emotional support from the partners 6 weeks after childbirth as well as the change in emotional support from the partner between 6 weeks and
17 months postpartum did not relate to the risk of ranking among the "nondepressive symptom" trajectory compared with the "improvement" trajectory.

Prediction of the depressive symptom trajectory membership by different exchange patterns of emotional support within the partnership

Binary logistic regressions were applied to assess how the different exchange patterns of emotional support 5 months after childbirth relate to the depressive symptoms trajectories. Belonging to the "bidirectional high supporters" 5 months postpartum was a significant predictor. Mothers who were living in a partnership with high levels of support were more than three times more likely to be in the "nondepressive symptom" trajectory than in the "stable depressive symptom" trajectory $(\mathrm{OR}=3.68 ; 95 \% \mathrm{CI}$, 1.19-11.39) compared to the reference category "bidirectional low supporters". For mothers ranking among the "providers" and "receivers" 5 months after childbirth, no significant relations for a depressive symptom trajectory membership were found in comparison with the "bidirectional low supporters" (all $P$ values $>0.145$ ) (not presented in a table). 


\section{Discussion}

The results of our study are based on a longitudinal design examining the mother's psychological adaptation to motherhood via the trajectories of depressive symptoms up to 17 months after childbirth. First, the study found that depressive symptom trajectories were related to the impact of emotional support mothers received from their partners in week 6 and the change in this support up to 17 months after childbirth. Among mothers with "stable depressive symptoms" lower levels of received emotional support from their partners at week 6 were reported compared to mothers with nondepressive symptoms. Importantly, mothers who experienced an increase in emotional support from their partners from 6 weeks up to 17 months after childbirth were much less likely to develop symptoms of depression even if they had reported depressive symptoms 5 months after childbirth. In contrast, women who reported a decremental change in partner support developed more symptoms of depression even when they reported no depressive symptoms 5 months postpartum. We were aware that our findings were in accordance with previous studies reporting that an emotionally supportive partner turned out to be a protective factor for the mother's prolonged psychological adjustment to motherhood after childbirth (Beck 2001; Boyce and Hickey 2005; Lemola et al. 2007; Rich-Edwards et al. 2006). However, because it was unclear if the perception of received emotional support from the partner for mothers with depressive mood seemed lower because of their depressive symptoms or if their depressive symptoms were a consequence of the low emotional support in our study, the male partners were also asked to evaluate the exchange of emotional support in their partnership similar to the mothers. The correlations between maternal and paternal report of the exchange of emotional support 5 months postpartum was moderately high.

In line with theories on the equity of human behavior and well-being (Walster et al. 1978), we found a broad association between the exchange of emotional support in the partnership and postpartum depressive symptoms. Belonging to the supporter group with high levels of both providing and receiving support 5 months after childbirth turned out to have a protective effect on the mother's depressive symptoms trajectories. In contrast, for mothers who belonged to the "bidirectional low supporter" group 5 months postpartum, the likelihood of suffering from depressive symptoms 12 months later was more than three times higher than for mothers who were living in a satisfying reciprocal supportive partnership. A comparable cross-sectional design study (Stevenson et al. 1999) reported that "bidirectionals" were less depressed than "low supporters". Our findings confirmed these results in a methodologically more appropriate longitudinal design and approached the question of the causal link between social support and depressive symptoms. In comparison, Gleason et al. (2003) has suggested that supportive interaction within couples depends on the duration of the demanding situation; particularly in demanding but probably temporary circumstances, equity in exchanging support may be less important than in chronically demanding situations.

In sum, receiving emotional support from the partner in a relationship with a satisfying exchange of reciprocal emotional support turned out to be a protective factor for the mother's prolonged psychological adjustment beyond the early postpartum period.

There are strengths and limitations to our study. The strengths are that our study follows a longitudinal design. Answering questions like ours would hardly be possible in a design that is cross sectional. Another strength is that our data are based on a representative sample of the population and that the results are therefore generalisable regarding postpartum depressive symptoms. A major limitation is that the investigation of psychological adaptation in mothers and their partners started 4 months earlier for the mothers than for their partners. It is possible that the support the mothers received from their partners 6 weeks after childbirth does not have the same relevance for the adjustment to parenthood as the support the partners received approximately 4 months later from their wives. Thus, the application of the support categories 5 months postpartum may introduce a possible perceptions bias. Another limitation was the selective attrition regarding postpartum depressive symptoms which were more common in unavailable mothers. It is difficult to assess the bias on our findings that could be introduced by these missing responses. Further, about $10 \%$ of the mothers with postpartum depressive symptoms in our study had EPDS scores between 10 and 12 and could possibly have been mislabeled as having a depressive episode. The psychological distress in the postpartum period proved to be complex and mothers who were affected with postpartum depressive symptoms could have been suffering from disabling anxiety or an adjustment disorder (Rowe et al. 2008). Additionally, the follow-up period from 5 up to 17 months after childbirth was long. With shorter measurement intervals a more differentiated picture of the trajectories of depressive symptoms might have been provided.

A supportive partnership can help mitigate stress during adjustment to motherhood and it has been found that good emotional support during pregnancy is a strong protective factor against depressive symptoms postpartum (Robertson et al. 2004). Intervention programs are an opportunity for women suffering from PPD and for their partners to become aware of a mother's psychosocial functioning after childbirth. Miseri et al. (2000) found that in treatments of 
postpartum depression in which the mothers' partners attended therapy sessions about supporting their wives, a significant decrease in depressive symptoms occurred. Depressive symptoms may be more persistent in women whose reciprocal emotional support with their partner is poor beyond the early postpartum period than has been previously assumed. Further studies should also focus on the partner's pre-birth support and on possible education programs concerning postpartum psychosocial care (Matthey et al. 2002) and their impact on his wife's psychological adjustment in this time period.

Acknowledgments This study was conducted within the research project "Substance use and psychosocial risk of mothers in Switzerland", which was supported by the Swiss Federal Office of Public Health (SFOPH Decree No 03.001623 to Prof. Dr. A. Grob). The study was run at the Department of Personality, Individual Differences and Diagnostics, University of Berne, and at the Department of Personality and Developmental Psychology, University of Basel. We thank Dr. Heather Murray for her invaluable help in proofreading the manuscript and above all the mothers and fathers who participated in this study.

\section{References}

Bandura A (1986) Social foundations of thought and action: a social cognitive theory. Prentice Hall, Englewood Cliffs, NJ

Beck CT (2001) Predictors of postpartum depression: an update. Nurs Res 50:275-285

Bergant AM, Nguyen T, Heim K, Ulmer H, Depunt O (1998) German translation and validation of the 'Edinburgh Postnatal Depression Scale' EPDS. Dtsch Med Wochenschr 123:35-40

Boyce Ph, Hickey A (2005) Psychosocial risk factors to major depression after childbirth. Soc Psychiatry Psychiatr Epidemiol 40:605-612

Cialdini RB, Darby BL, Vincent JE (1973) Transgression and altruism: a case for hedonism. J Exp Soc Psychol 9:502-516

Cohan S, Wills TA (1985) Stress, social support, and the buffering hypothesis. Psychol Bull 98:310-357

Cox MJ, Owen MT, Lewis JM, Henderson VK (1989) Marriage, adult adjustment, and early parenting. Child Dev 60:1015-1024

Cox JL, Murray D, Chapman G (1993) A controlled study of the onset, duration and prevalence of postnatal depression. B J Psychiatry 163:27-31

Cox JL, Chapman G, Murray D, Jones P (1996) Validation of the Edinburgh Postnatal Depression Scale (EPDS) in non-postnatal women. J Affect Disord 39:185-189

Cox JL, Holden JM, Sagovsky R (1998) Detection of postnatal depression. Development of the 10-item Edinburgh Postnatal Depression Scale. Br J Psychiatry 150:782-786

Drack G, Ackermann-Liebrich U, Schindler C (1998) Totgeburten und Säuglingssterblichkeit in der Schweiz 1986-1992. Bundesamt Stat

Eberhard-Gran M, Eskild A, Tambs K, Samuelsen SO, Opjordsmoen $S$ (2002) Depression in postpartum and non-postpartum women: prevalence and risk factors. Acta Psychiatr Scand 106:426-433

Gleason MEJ, Iida M, Bolger N, Shrout PE (2003) Daily supportive equity in close relationships. Pers Soc Psychol Bull 29:10361045
Goodman JH (2004) Postpartum depression beyond the early postpartum period. J Obstet Gynecol Neonatal Nurs 33:410-420

Lee D, Yip A, Chui H, Chung T (2002) Screening for postnatal depression using the double-test strategy. Psychosom Med 62:258-261

Lemola S, Grob A (2008) Smoking cessation during pregnancy and relapse after childbirth: the impact of the grandmother's smoking status. Matern Child Health J 12:525-533

Lemola S, Stadlmayr W, Grob A (2007) Maternal adjustment five months after birth: the impact of the subjective experience of childbirth and emotional support from the partner. J Reprod Infant Psychol 25:190-202

Life Course and Health Research Center (2001) Transitions: a study of stress and well-being in young adulthood. Wave II questionnaire. Drug use trajectories ethnic/racial comparisons. http://www.fiu.edu/ lchrc/pdfs/w2instrument.pdf. Accessed 22 May 2006

Maton KI (1987) Patterns and psychological correlates of material support within a religious setting: the bidirectional support hypothesis. Am J Community Psychol 15:185-207

Matthey S, Morgan M, Healey L, Barnett B, Kavanagh DJ, Howie P (2002) Postpartum issues for expectant mothers and fathers. J Obstet Gynecol Neonatal Nurs 31:428-435

Merchant DC, Affonso DD, Mayberry LJ (1995) Influence of marital relationship and child-care stress on maternal depression symptoms in the postpartum. J Pediatr Obstet Gynaecol 16:193-200

Miseri S, Kostaras X, Fox D, Kostaras D (2000) The impact of partner support in the treatment of postpartum depression. Can J Psychiatry 45:554-558

O'Hara MW, Swain A (1996) Rates and risk of postpartum depression: a meta-analysis. Int Rev Psychiatry 8:37-54

Patel V, Rodrigues M, DeSouza N (2002) Gender, poverty, and postnatal depression: a study of mothers in Goa, India. Am J Psychiatry 159:43-47

Rich-Edwards JW, Kleinman K, Abrams A et al (2006) Sociodemographic predictors of antenatal and postpartum depressive symptoms among women in a medical group practice. J Epidemiol Community Health 60:221-227

Robertson E, Grace S, Wallington T, Stewart DE (2004) Antenatal risk factors for postpartum depression: a synthesis of recent literature. Gen Hosp Psychiatry 26:289-295

Rohde A (2001) Die vielen Gesichter der Depression. UNI-MED Verl, Bremen

Rowe HJ, Loh JR, Loh WM (2008) The Edinburgh Postnatal Depression Scale detects but does not distinguish anxiety disorders from depression in mothers of infants. Arch Womens Ment Health 11:103-108

Schmidt CO (2008) Risk quantification in epidemiologic studies. Int J Public Health 53:118-119

Stevenson W, Maton KI, Teti DMS (1999) Social support, relationship quality, and well-being among pregnant adolescents. J Adolesc 22:109-121

Swiss Federal Office of Statistics (2005a) Medizinische Statistik der Krankenhäuser. Available via Medienmitteilung Nr. 0350-050740

Swiss Federal Office of Statistics (2005b) Current population and population growth—key data: births. http://www.bfs.admin.ch. Assessed 3 September 2006

Swiss Federal Office of Statistics (2005c) Swiss labour force statistics (SAKE). Je-d-03.02.0112.xls. http://www.bfs.admin.ch. Assessed 3 September 2006

Walster E, Walster GW, Berscheid E, Austin W (1978) Equity: theory and research. Allyn and Bacon, Boston 